\title{
Hospital care for primary hyperparathyroidism in Italy: a 6-year register-based study
}

\section{Cristiana Cipriani, Vincenzo Carnevale', Federica Biamonte, Sara Piemonte, Jessica Pepe, Luciano Nieddu², John P Bilezikian ${ }^{3}$ and Salvatore Minisola}

Department of Internal Medicine and Medical Disciplines, 'Sapienza' University of Rome, Viale del Policlinico 155 00161 Rome, Italy, 'Unit of Internal Medicine, 'Casa Sollievo della Sofferenza' Hospital, IRCCS, Viale dei Cappuccini snc, 71013 San Giovanni Rotondo, Foggia, Italy, ${ }^{2}$ Faculty of Economics (L.N.), LUSPIO University, Via delle Sette Chiese 139, 00145 Rome, Italy and ${ }^{3}$ Metabolic Bone Diseases Unit, Division of Endocrinology, Department of Medicine, College of Physicians and Surgeons, Columbia University, 630 West 168th Street, New York, New York 10032, USA
Correspondence should be addressed to C Cipriani

Email

cristianac@alice.it

\begin{abstract}
Objective: Primary hyperparathyroidism (PHPT) is one of the most frequently diagnosed endocrine disorders, but few studies have focused on hospital management of the disease in Europe. We investigated the frequency of hospital admission for diagnosis and surgical treatment of PHPT in Italy.

Design: A retrospective study was conducted for investigating the hospital care for PHPT in Italy.

Methods: We retrieved data from the 'Record of Hospital Discharge' of the Italian Health Ministry, from 2006 to 2011, and analyzed the codes corresponding to PHPT-related diagnoses and surgical procedures.

Results: Overall, 46275 hospitalization episodes for PHPT were identified during the entire period (69\% in women and $31 \%$ in men; mean age $63.3 \pm 39.8$ years). Patients' mean age significantly increased during the years $(P<0.001)$. The mean length of stay was $8.2 \pm 10.5$ days ( $28 \%$ of the episodes requiring $<3$ days of stay). Admissions for surgical procedures were 12457 accounting for $26.9 \%$ of the total hospitalizations. There was a trend to a significant increase in the percentage of surgery $(P<0.05)$. The mean hospitalization rate for PHPT was $12.9 / 100000$ inhabitants per year and the trend showed a significant decrease during the period of 2006-2011 $(P<0.0001)$. The mean hospitalization rate for PHPT surgery was 3.65/100 000 per year, which significantly increased over time $(P<0.001)$.

Conclusions: PHPT considerably influences the Italian Hospital healthcare system. We observed a tendency to a decrease in the frequency of hospitalization during the period of 2006-2011, most probably because of economic issues, a concomitant increased age of patients, and, interestingly, also a progressive increase in the percentage of surgical treatment among patients admitted for PHPT.
\end{abstract}

\section{Introduction}

Primary hyperparathyroidism (PHPT) is one of the most common endocrine disorders worldwide. As the clinical presentation of the disease has evolved over the past decades from the symptomatic variant through the asymptomatic variant and now even to a normocalcemic variant, the epidemiology of PHPT has undergone profound changes $(1,2,3,4)$. These studies have shown that the incidence of the disease is closely related to the frequency of routinely obtained biochemical screening tests, socioeconomic issues (i.e. low vitamin D status), dietary habits (i.e. calcium and vitamin D supplementation), as well as the degree of awareness among physicians $(2,3)$. Not unexpectedly, these varying presentations are associated with different and countryspecific epidemiological patterns $(1,2,3,5)$. The Rochester study, for example, showed an incidence of PHPT of 15.7 per 100000 in 2001, representing a progressive decline since 1974 (3). From United States classification codes, the 
rate in 1999 was 1.8 per 100000 inhabitants per year, also representing a progressive decline from 1977 to 1999 (2).

To date, few studies have focused on the epidemiology and hospital management of the disease in Europe. Our group showed a prevalence of PHPT of 15 out of 286 women (5.2\%) and two out of 143 men (1.4\%) based upon referrals to the Metabolic Bone Disease Unit in Rome, Italy, from 2007 to 2009 (6). Similar results were reported in another Italian study, where a $4.5 \%$ prevalence of PHPT was observed among 1095 patients consecutively admitted for reduced bone mineral density (BMD) and/or for history of fragility fracture (7). Misiorowski and Zgliczyński (8) reported an $11.5 \%$ prevalence of PHPT among subjects with low bone mass in Poland. Data from a 10-year population-based study in Tayside, Scotland, showed an increasing prevalence of PHPT (from 1.82 to 6.72 per 1000 adult population), as well as cyclicity in its annual incidence (9). As far as hospital management is concerned, Richert et al. (5) reported a hospitalization rate for PHPT in Switzerland, from 2000 to 2004, of 8.3 per 100000 , significantly higher in those who are at the age of $\geq 50$ years and in women. Among those hospitalized for PHPT, the rate of surgically treated patients was $45.2 \%$, with a higher proportion in those aged $<50$ years $(81.3 \%)$ (5). The Danish study, which included all patients diagnosed with PHPT in the hospital discharge registry from 1977 to 2010, showed an annual rate of 16 per 10000 and a continuing increase during the period of interest, with a fluctuation in the year-to-year incidence (10).

To our knowledge, no study to date has evaluated the prevalence of PHPT among hospitalized patients in Italy. We therefore performed a 6-year register-based study aimed at investigating the frequency of hospital admissions for the diagnosis and surgical treatment of PHPT in Italy. Additionally, we evaluated the frequency of associated skeletal and renal complications.

\section{Subjects and methods}

This retrospective study is based on the data from the registry of the Italian Health Ministry, from 2006 to 2011, when the Italian version of the ICD-9 CM classification system was instituted (www.salute.gov). We analyzed the codes corresponding to the following medical diagnoses: 'PHPT' (252.01), 'not specified hyperparathyroidism' (252.00), non-tumor-related 'hypercalcemia' (275.42), 'benign parathyroid neoplasia' (227.1), malignant parathyroid neoplasia (194.1), 'multiple endocrine neoplasia' (MEN) (258.0), as well as the surgical procedures, including 'complete parathyroidectomy'
(06.81), 'other parathyroidectomies' (06.89), and 'other parathyroid procedures' (06.99). From one to five secondary-listed diagnoses were taken into account. Cases of non-tumor-related 'hypercalcemia' were retrieved by considering 'hypercalcemia' as the diagnosis of interest, excluding cases where malignancy was found to be associated. This diagnosis was added in order to avoid underestimation of the results, as many cases of PHPT in Italy are diagnosed as 'hypercalcemia' and not associated with malignancy at discharge, particularly when the complete results of the diagnostic workup are not available.

All data were retrieved from the archive of the 'Record of Hospital Discharge' ('Scheda di Dimissione Ospedaliera', SDO). The SDO is the most valuable instrument for the collection of the information on each patient discharged from public and private hospital institutions all over the country (www.salute.gov). It is mandatory both for hospitalization and 1-day hospital admission (www.salute.gov). Information, collected according to the local privacy laws, were filled in by the physician in charge of the patient at discharge and include clinical (diagnoses, symptoms, surgery, diagnostic/therapeutic procedures, and type of discharge) and organizational aspects of hospitalization (www.salute.gov).

Among all the variables available in the SDO, the following were considered: regional code (region of hospitalization), sex, age, date of hospitalization, date of discharge, and days of hospitalization of the patient. Moreover, to have an overall idea on how PHPT influences hospital care costs in Italy, an evaluation of the diagnosisrelated groups (DRGs) associated with the aforementioned diagnoses was performed. The DRGs represent groups of diagnoses related to each other as far as their costs are concerned (www.salute.gov). The classification of any hospitalization event through the DRG, performed by the physician in charge of the patient, is therefore also aimed at categorizing the rate for the service carried out by the hospital (www.salute.gov). The following DRGs were considered: 'parathyroid surgery' (289), 'thyroid surgery' (290), 'other surgeries for endocrine, nutritional, and metabolic diseases with complications' (292), 'other surgeries for endocrine, nutritional, and metabolic diseases without complications' (293), 'nourishment and miscellaneous metabolic disorders, age $>17$ years, with complications' (296), 'nourishment and miscellaneous metabolic disorders, age $>17$ years, without complications' (297), 'endocrine disorders with complications' (300), and 'endocrine disorders without complications' (300). 
Moreover, diagnoses/procedures associated with renal and skeletal complications were analyzed, as follows: for renal involvement 'calculus in kidney' (592.0), 'calculus in ureter' (592.1), 'calculus in bladder' (594.0 and 594.1), 'calculus in urethra' (594.2), 'other lower urinary tract calculi' (594.8 and 594.9), 'urinary calculus, unspecified' (592.9), and 'lithotripsy' (59.95); and for skeletal involvement: 'osteoporosis' (733.0), 'pathological fracture of humerus' (733.11), 'pathological fracture of distal radius and ulna' (733.12), 'pathological fracture of vertebrae' (733.13), 'pathological fracture of neck of femur' (733.14), 'pathological fracture of other specified parts of femur' (733.15), 'pathological fracture of tibia or fibula' (733.15), 'fracture of humerus' (812), 'fracture of clavicle' (810), 'fracture of neck of femur' (820), 'fracture of radius and ulna' (813), 'fracture of pelvis' (808), and 'other, multiple, and ill-defined fractures of lower limb (827). As for the latter diagnoses, and as already noted, any diagnosis of malignancy was previously excluded.

\section{Statistical analysis}

The total number of hospitalizations for PHPT throughout the 6-year period, as well as its trend over time, was evaluated. Demographic data, such as sex and age, were considered. Patients' mean age is reported as mean \pm s.D. and the trend over time was evaluated.

The hospitalization rate was calculated using the following formula: (diagnoses/mean annual resident population) $\times 100000$ inhabitants, by considering the total number of inhabitants in the country for any year, as indicated by the Italian National Statistics Institute database (available online at http//www.istat.it). The trend of the hospitalization rate over the 6 years was calculated for all the diagnoses and by dividing for surgical and medical diagnoses. The total number of DRGs and the ratio DRG/total diagnoses was calculated, by also dividing between medical and surgical DRGs and their trend over the observation period was evaluated.

Any of the aforementioned trends was calculated by the test for equality of proportions without continuity correction.

To study the effect of time (in years) and type of diagnoses (medical/surgical) on the duration of hospitalizations, a zero-truncated Poisson regression model has been fit to the data considering both effects and their interactions.

To test whether there was any effect of time and type of diagnoses on the incidence of short hospitalizations
( $<3$ days), a logistic model was used, considering both simple and interaction effects.

Finally, the total number of kidney and skeletal complications and the ratio complications/total diagnoses were calculated. $P$ values $<0.05$ were considered significant and the $\mathrm{R}$ package (version 3.02) was used for statistical calculations.

\section{Results}

Table 1 summarizes the number of hospitalization episodes, demographic data, and the length of stay during the 6 years of observation.

Overall, 46275 hospitalization episodes for PHPT were identified during the entire period, the mean number being 7712/year. There was a significant decrease in the number of hospitalizations for PHPT during the period of 2006-2011 (Table 1, $P<0.05$ ). Among those hospitalizations, $69 \%$ were in women and $31 \%$ in men (female/ male ratio 2.23:1; mean age $63.3 \pm 39.8$ years). Patients' mean age significantly increased during the 6 years $(P<0.001$, Table 1$)$. The mean length of stay was $8.2 \pm$ 10.5 days ( $28 \%$ of the episodes requiring $<3$ days of stay). The overall number of admissions for surgical procedures was 12457 accounting for $26.9 \%$ of total hospitalizations over the 6-year period. There was a significant trend to increase the percentage of admissions for surgical procedures during the period $(25.7 \%$ in $2006,26.6 \%$ in $2007,26.8 \%$ in $2008,27.9 \%$ in $2009,26.9 \%$ in 2010 , and $27.6 \%$ in $2011 ; P<0.05)$.

In $61.1 \%$ of the cases, the PHPT-related diagnosis/ procedure was the first-listed diagnosis/procedure, as was

Table 1 Total number of the hospitalization episodes, demographic data, and the length of stay during the period of observation.

\begin{tabular}{|c|c|c|c|c|}
\hline Year & $\begin{array}{c}\text { Number of } \\
\text { hospitalization } \\
\text { episodes }^{a}\end{array}$ & $\begin{array}{c}\text { Sex } \\
\text { (F:M ratio) }\end{array}$ & $\begin{array}{c}\text { Age (years) } \\
(\text { mean } \pm \text { s.D. })^{b}\end{array}$ & $\begin{array}{c}\text { Days of stay } \\
\text { (mean } \pm \text { s.D.) }\end{array}$ \\
\hline 2006 & 7715 & $2.24: 1$ & $62.7 \pm 37.5$ & $8.1 \pm 10$ \\
\hline 2007 & 7454 & $2.32: 1$ & $64.9 \pm 58.7$ & $8.1 \pm 10.2$ \\
\hline 2008 & 7809 & $2.26: 1$ & $63.2 \pm 41.7$ & $8.1 \pm 10$ \\
\hline 2009 & 7876 & $2.26: 1$ & $63.3 \pm 40.1$ & $8.1 \pm 9.9$ \\
\hline 2010 & 7899 & 2.19:1 & $63.3 \pm 32.3$ & $8.3 \pm 10.9$ \\
\hline 2011 & 7522 & 2.09:1 & $65.8 \pm 18.6$ & $8.1 \pm 11.8$ \\
\hline 2006-2011 & 46275 & $2.23: 1$ & $63.3 \pm 39.8$ & $8.2 \pm 10.5$ \\
\hline
\end{tabular}

F:M, female:male.

a Significant trend: $P<0.05$ by test for equality of proportions without continuity correction.

${ }^{b}$ Significant trend: $P<0.001$ by test for equality of proportions without continuity correction. 


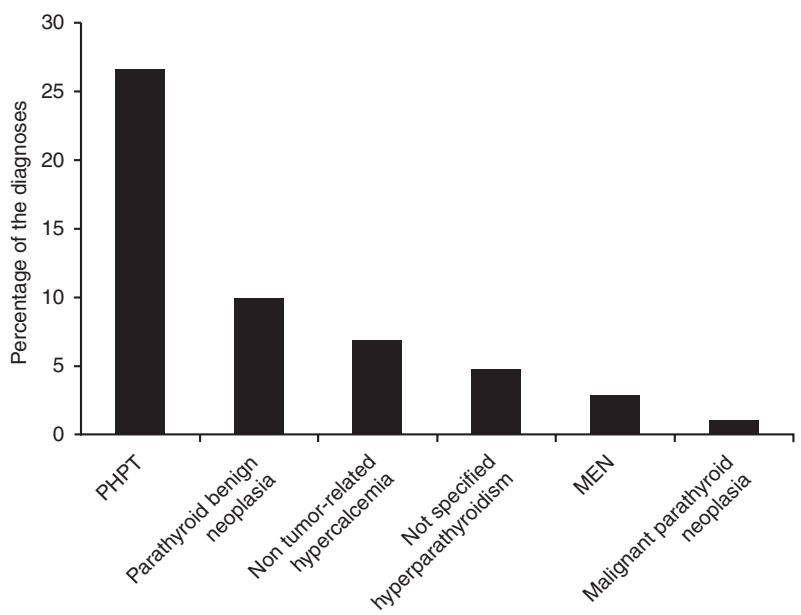

Figure 1

Percentage of cases of PHPT-related diagnoses over the total hospitalization episodes.

the corresponding DRG. The percentage of the first-listed diagnoses over the total cases followed in this order: 'PHPT' 26.6\%, 'benign parathyroid neoplasia' 9.9\%, nontumor-related 'hypercalcemia' $6.9 \%$, 'not specified hyperparathyroidism' $4.8 \%$, 'MEN' $2.8 \%$, and 'malignant parathyroid neoplasia' 1\% (Fig. 1); 'other parathyroidectomy' 23\%, 'complete parathyroidectomy' $0.8 \%$, and 'other parathyroid procedures' $0.4 \%$.

The analysis of the DRG codes showed that $32.8 \%$ of all the cases were assigned to a medical DRG, while $28.3 \%$ were assigned to a surgical one. The trend for the medical DRGs over time showed a significant reduction (from $34.8 \%$ in 2006 to $30.6 \%$ in 2011 , Table $2 ; P<0.0001$ ), while there was an increase in the percentage of the surgical DRGs (from $27.4 \%$ in 2006 to $28.3 \%$ in 2011, Table 2; $P<0.01)$. Interestingly, among all the hospitalization events for which a PHPT-related DRG was assigned, we observed a significant trend to an increase in the mean length of stay for medical DRGs (from $6.1 \pm 7.4$ in 2006 to $6.7 \pm 6.8$ in $2011 ; P<0.001)$ and to a decline for surgical DRGs (from $5 \pm 6.2$ in 2006 to $4.4 \pm 6$ in $2011 ; P<0.001$ ). Concomitantly, there was an increase in the percentage of short-length hospitalization episodes $(<3$ days of stay; from $13.2 \%$ in 2006 to $18.8 \%$ in $2011 ; P<0.001$ ) as far as surgical DRGs are concerned, while short hospitalizations with medical DRGs showed a decline (from 18.7\% in 2006 to $13.7 \%$ in $2011 ; P<0.001)$.

Overall, considering all the SDOs where PHPT-related diagnostic codes were listed as either the first or other diagnoses, the mean hospitalization rate for PHPT was 12.9/100 000 inhabitants per year and the trend showed a significant decrease during the period of 2006-2011 (Fig. 2A; $P<0.0001$ ). The mean hospitalization rate for PHPT surgery was 3.65/100 000 per year, significantly increasing over the period of observation (from $3.60 / 100000$ per year in 2006 to 3.62 in 2011; $P<0.001$; Fig. 2B). The mean hospitalization rate for PHPTassociated DRGs was 7.9/100 000 inhabitants per year.

The total number of diagnoses of kidney stones associated with PHPT diagnoses was 1902 over the period of 2006-2011 amounting to $4.1 \%$ of the cases. The majority of the cases of kidney stones were secondarylisted diagnoses (77.6 vs $22.4 \%$ as first-listed diagnoses) and were diagnosed in women (F:M ratio 2:1). Lithotripsy was associated with $2.8 \%$ of all kidney stone diagnostic classifications.

The rate of diagnoses of osteoporosis/fragility fractures associated with PHPT diagnoses was $4.6 \%$ over the entire period $(86 \%$ as secondary and $14 \%$ as first-listed diagnoses; F:M ratio 4.1:1). The most common diagnoses were 'osteoporosis' (72.3\% of all the skeletal complications) and 'pathological fracture of vertebrae' (22.9\%).

The mean age for skeletal-related complications $(66.4 \pm 12.5)$ was higher than the mean age of kidney stones complications $(55.5 \pm 17.7)(P<0.0001)$.

\section{Discussion}

Since the dramatic rise in the frequency of PHPT as a diagnostic entity with the introduction of the multichannel automated analysis in the 1970s, the epidemiology of the disease has substantially changed (3). To our knowledge, this is the first study to investigate the incidence and trends in PHPT diagnoses in Italy over a long period of time. Our data show that PHPT is prevalent among hospitalized patients in Italy and that women predominate over men. Women also show a higher rate of kidney and skeletal complications than men.

Table 2 Percentages of DRG in the 6 years of observation.

\begin{tabular}{lccc}
\hline Year & & Medical DRG $^{\mathrm{a}}(\%)$ & Surgical DRG $^{\mathrm{b}}(\%)$ \\
\hline 2006 & 34.8 & 27.4 \\
2007 & 34.7 & 28 \\
2008 & 32.4 & 28.1 \\
2009 & 32.4 & 29.1 \\
2010 & 31.7 & 27.8 \\
2011 & 30.6 & 29.2 \\
$2006-2011$ & 32.8 & 28.3 \\
\hline
\end{tabular}

DRG, diagnosis-related group.

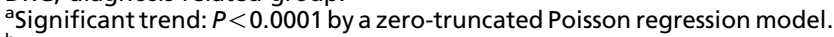
${ }^{b}$ Significant trend: $P<0.01$ by a zero-truncated Poisson regression model. 


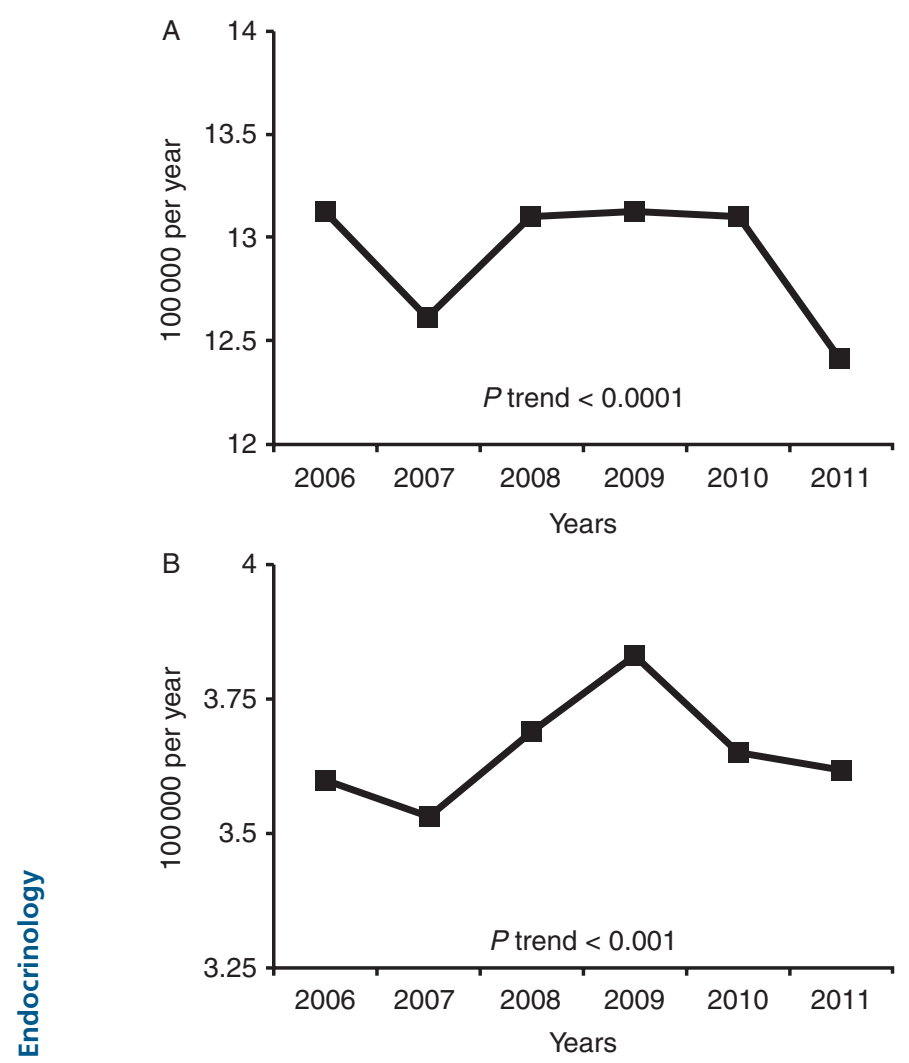

Figure 2

(A) Hospitalization rate for PHPT/100 000 per year in Italy (only the first-listed diagnoses). (B) Hospitalization rate for PHPT surgery/100 000 per year in Italy (only the first-listed diagnoses).

We found a mean hospitalization rate of 12.9/100 000 inhabitants per year for the period of 2006-2011, thus demonstrating a higher frequency of PHPT diagnoses among inpatients, compared with other studies $(2,5,11,12)$. It is interesting to note that when the mean hospitalization rate for any disease in Italy in the same period is calculated (12 183/100 000 inhabitants per year; data available at www. salute.gov), PHPT represented almost $1 / 1000$ of all causes of hospitalization. Data on the hospitalization rate for PHPT-associated DRGs (7.9/100 000 inhabitants per year) substantially confirm this finding and give more accurate information on how hospital care for PHPT represents a finite component of all Italian health-related public costs. In our study, the PHPT-related DRG was assigned to more than half $(61 \%)$ of the total number of hospitalizations (46 275), thus confirming that PHPT considerably impacts the Italian Hospital healthcare system.

The trend of the hospitalization rate for PHPT, showing a significant reduction over the years (Fig. 2A), is in apparent contradiction to Abood and Vestergaard
(10), where an increase in the rate of PHPT in both women and men was reported from 1990-1998 to 2010 in Denmark. The different results could be ascribed to differences in the population studied, as well as in the length of the period analyzed (between 12 and 20 years in the Danish experience and 6 years in our study), particularly considering that Abood and Vestergaard (10) described large fluctuations in the incidence from 1 year to the next.

Data about surgery also deserve discussion. We observed that the mean proportion of admission for surgery was lower than the Swiss figure of $45.2 \%$ (5) and the US experience (12000 parathyroidectomies are reported over 22000 diagnoses of PHPT in 1999 (2, 11)). However, along with the overall reduction in hospitalization for PHPT, the proportion of surgically treated patients significantly increased over years (Fig. 2B). Interestingly, age progressively increased over the same period. Taken together, these data do not confirm a real reduction in PHPT incidence, but rather a trend to a change in the scenario of patients hospitalized for PHPT in Italy in the last years. Our results therefore show that in-hospital admission for PHPT, unlike other countries, represents an important aspect of evaluation for this disorder. This point leads to several others that are worthy of comment. It should be emphasized that $28 \%$ of the total hospitalization episodes were $<3$ days of length. This finding is important to note, as those cases actually represent patients admitted for suspected PHPT, particularly among specialized centers, for a 1- or 2-day hospitalization. The way those patients are managed is therefore very close to what is usually observed among outpatients and this point should be addressed in interpreting the profile of patients hospitalized for PHPT in Italy. These short-stay admissions also include hospitalizations for PHPT surgery. Interestingly, our data showed a tendency for a reduction in the proportion of patients hospitalized for PHPT evaluation in the short period, while the mean length of stay of surgical patients declined and the proportion of short-term hospitalizations ( $<3$ days) for surgery significantly increased over time. On the other hand, the overall length of stay ( $8.2 \pm 10.5$ days) also indicates that PHPT management is still considered a challenging task by most Italian physicians outside referral centers. It also suggests that the in-hospital diagnosis of PHPT in many cases occurs after the serum calcium is identified as a component of the routine laboratory workup of inpatients in Italy.

Multiple factors should be taken into account in evaluating our findings. First, the high proportion of 
elderly people in Italy could in part account for the increased mean age of PHPT inpatients. Moreover, the tendency to restrict public outlays, including those related to the healthcare system, has contributed to the aforementioned changes. Data from the Italian Health Ministry indeed showed a tendency to a reduction in the overall hospitalization rate in the period of 2006-2011 (from $13471 / 100000$ inhabitants per year in 2006 to $10978 / 100000$ inhabitants per year in 2011; www. salute.gov). Even if it is still to a lesser extent than in other countries $(2,5,11)$, PHPT patients (particularly the older ones) are currently mostly hospitalized for surgery in Italy, for which the length of stay has significantly reduced in the last years. This point could also account for a higher proportion of minimally invasive surgical procedures.

Analysis of the distribution of first-listed diagnoses showed, again, different results compared with other countries, particularly a higher rate of hospitalization in our experience (7.9/100 000/year vs $1.8 / 100$ 000/year in USA in 1999) (11). Moreover, we observed an interesting pattern as far as the prevalence of the more rare forms of the disease is concerned (Fig. 1). The prevalence of multiple endocrine neoplasia and parathyroid carcinoma in our cohort (Fig. 1) substantially reflected previous epidemiological findings $(12,13,14,15)$ and suggested that probably a large proportion of those rare causes of PHPT are in-hospital managed in Italy. Different findings were instead observed as far as the classical complications of the disease are concerned. Our data showed a lower rate of kidney stones and skeletal complications among patients hospitalized for PHPT compared with previous reports $(16,17,18,19)$. This finding suggests that the vast majority of patients hospitalized for PHPT in Italy have no overt complications of the disease. Differences between this and other studies could be related to different populations and sources of data, as only inpatients were considered in our study. The last point could therefore represent a limit for kidney and skeletal evaluation, which actually did not represent the main outcome of the study. However, it should be noted that skeletal and kidney complications are not routinely observed in asymptomatic PHPT patients, regardless of the medical setting in which they are managed. Kidney stones were, however, more prevalent among younger patients, as observed in other studies $(20,21)$, while patients with concomitant osteoporosis/fragility fractures were older. Hence, the possibility that the high prevalence of elderly patients in our cohort could have influenced the lower rate of kidney stones we observed compared with other reports should be taken into account. In accordance with previous reports, our data showed a higher prevalence of vertebral fractures in PHPT hospitalized patients, compared with other skeletal sites $(18,22,23)$. This finding further confirms the involvement of trabecular bone in PHPT, possibly related to perturbations of the micro-architectural aspects (24).

In conclusion, PHPT influences the Italian Hospital healthcare system. The disease is particularly prevalent among women in their sixties with the majority of patients hospitalized for PHPT showing no clear complications. Mostly because of economic issues, we observed a tendency to a reduction in the frequency of hospitalization during the period of 2006-2011, as well as a concomitant increase in the age of the patients, and, interestingly, a progressive increase in the percentage undergoing surgery. Higher in-hospital prevalence of PHPT over a 6-year period of observation was found compared with other countries. A number of factors possibly influencing the epidemiology, as well as the pattern of clinical presentation of PHPT, should be taken into account in evaluating those discordant results. To date, there are no formal studies exploring the modalities used to characterize the clinical presentation of PHPT in Europe and a significant proportion of cases are not even diagnosed or treated (25). Differences among studies therefore point out the need for further focused research to better define the epidemiology and the clinical presentation of PHPT in different geographical areas.

\section{Declaration of interest}

The authors declare that there is no conflict of interest that could be perceived as prejudicing the impartiality of the research reported.

\section{Funding}

This research did not receive any specific grant from any funding agency in the public, commercial or not-for-profit sector.

\section{Author contribution statement}

C Cipriani contributed to the design of the work, acquisition, analysis, and interpretation of data and to manuscript drafting. V Carnevale contributed to the design of the work and interpretation of data and revised the paper. F Biamonte, S Piemonte, and J Pepe contributed to the acquisition of data. $\mathrm{L}$ Nieddu contributed to data analysis. J P Bilezikian contributed to interpretation of data and revised the manuscript. $\mathbf{S}$ Minisola contributed to project ideation and interpretation of data, and revised the manuscript. All the authors have approved the final version of the manuscript.

\section{Acknowledgements}

The authors wish to thank Dr Flavia Carle and Dr Pietro Granella for their contribution in retrieving data from the Italian Health Ministry database. 


\section{References}

1 Wermers RA, Khosla S, Atkinson EJ, Hodgson SF, O'Fallon WM \& Melton LJ. The rise and fall of primary hyperparathyroidism: a population-based study in Rochester, Minnesota, 1965-1992. Annals of Internal Medicine 1997126 433-440. (doi:10.7326/0003-4819-126-6199703150-00003)

2 Clarke BL. Epidemiology of primary hyperparathyroidism. Journal of Clinical Densitometry 201316 8-13. (doi:10.1016/j.jocd.2012.11.009)

3 Wermers RS, Khosla S, Atkinson EJ, Achenbach SJ, Oberg AN, Grant CS \& Melton LJ. Incidence of primary hyperparathyroidism in Rochester, Minnesota, 1993-2001: an update on the changing epidemiology of the disease. Journal of Bone and Mineral Research 200621 171-177. (doi:10.1359/JBMR.050910)

4 Cusano NE, Maalouf NM, Wang PY, Zhang C, Cremers SC, Haney EM, Bauer DC, Orwoll ES \& Bilezikian JP. Normocalcemic hyperparathyroidism and hypoparathyroidism in two community-based nonreferral populations. Journal of Clinical Endocrinology and Metabolism 201398 2734-2741. (doi:10.1210/jc.2013-1300)

5 Richert L, Trombetti A, Herrmann FR, Triponez F, Meier C, Robert JH \& Rizzoli R. Age and gender distribution of primary hyperparathyroidism and incidence of surgical treatment in a European country with a particularly high life expectancy. Swiss Medical Weekly 2009139 400-404. (doi:smw-12635)

6 Romagnoli E, Del Fiacco R, Russo S, Piemonte S, Fidanza F, Colapietro F, Diacinti D, Cipriani C \& Minisola S. Secondary osteoporosis in men and women: clinical challenge of an unresolved issue. Journal of Rheumatology 201138 1671-1679. (doi:10.3899/jrheum.110030)

7 Eller-Vainicher C, Zhukouskaya VV, Morelli V, Palmieri S, Scillitani A, Beck-Peccoz P \& Chiodini I. Prevalence of subclinical contributors to low bone mineral density and/or fragility fracture. European Journal of Endocrinology 2013169 225-237. (doi:10.1530/EJE-13-0102)

8 Misiorowski W \& Zgliczyński W. Prevalence of primary hyperparathyroidism among patients with low bone mass. Advances in Medical Sciences 201257 308-313. (doi:10.2478/v10039-012-0062-2)

9 Yu N, Donnan PT, Murphy MJ \& Leese GP. Epidemiology of primary hyperparathyroidism in Tayside, Scotland, UK. Clinical Endocrinology 200971 485-493. (doi:10.1111/j.1365-2265.2008.03520.x)

10 Abood A \& Vestergaard P. Increasing incidence of primary hyperparathyroidism in Denmark. Danish Medical Journal 201360 A4567.

11 Popovic JR. National hospital discharge survey: annual summary with detailed diagnosis and procedure data. DHHS Publication No. (PHS)2001-1722. Hyattsville, MD: U.S. Department of Health and Human Services, 1999.

12 Sharretts JM \& Simonds WF. Clinical and molecular genetics of parathyroid neoplasms. Best Practice \& Research. Clinical Endocrinology \& Metabolism 201024 491-502. (doi:10.1016/j.beem.2010.01.003)

13 Marcocci C, Cetani F, Rubin MR, Silverberg SJ, Pinchera A \& Bilezikian JP. Parathyroid carcinoma. Journal of Bone and Mineral Research 200823 1869-1880. (doi:10.1359/jbmr.081018)
14 Pepe J, Cipriani C, Pilotto R, De Lucia F, Castro C, Lenge L, Russo S, Guarnieri V, Scillitani A, Carnevale V et al. Sporadic and hereditary primary hyperparathyroidism. Journal of Endocrinological Investigation 201134 (Suppl) 40-44.

15 Muhr C, Ljughall S, Akerstrom G, Palmer M, Bergstrom K, Enoksson P, Lundqvist $\mathrm{G} \&$ Wide L. Screening for multiple endocrine neoplasia syndrome (type 1) in patients with primary hyperparathyroidism. Clinical Endocrinology 198420 153-162. (doi:10.1111/j.1365-2265. 1984.tb00070.x)

16 Wu B, Haigh PI, Hwang R, Ituarte PH, Liu IL, Hahn TJ \& Yeh MW. Underutilization of parathyroidectomy in elderly patients with primary hyperparathyroidism. Journal of Clinical Endocrinology and Metabolism 201095 4324-4330. (doi:10.1210/jc.2009-2819)

17 Starup-Linde J, Waldhauer E, Rolighed L, Mosekilde L \& Vestergaard P. Renal stones and calcifications in patients with primary hyperparathyroidism: associations with biochemical variables. European Journal of Endocrinology 2012166 1093-1100. (doi:10.1530/ EJE-12-0032)

18 De Geronimo S, Romagnoli E, Diacinti D, D’Erasmo E \& Minisola S. The risk of fractures in postmenopausal women with primary hyperparathyroidism. European Journal of Endocrinology $2006 \mathbf{1 5 5}$ 415-240. (doi:10.1530/eje.1.02225)

19 Silverberg SJ, Shane E, de la Cruz L, Dempster DW, Feldman F, Seldin D, Jacobs TP, Siris ES, Cafferty M, Parisien MV et al. Skeletal disease in primary hyperparathyroidism. Journal of Bone and Mineral Research 1989 4 283-291. (doi:10.1002/jbmr.5650040302)

20 Mollerup CL, Vestergaard P, Frøkjaer VG, Mosekilde L, Christiansen P \& Blichert-Toft M. Risk of renal stone events in primary hyperparathyroidism before and after parathyroid surgery: controlled retrospective follow up study. BMJ 2002325 807-810. (doi:10.1136/ bmj.325.7368.807)

21 Rejnmark L, Vestergaard P \& Mosekilde L. Nephrolithiasis and renal calcifications in primary hyperparathyroidism. Journal of Clinical Endocrinology and Metabolism 201196 2377-2385. (doi:10.1210/ jc.2011-0569)

22 Vestergaard P, Mollerup CL, Frøkjaer VG, Christiansen P, Blichert-Toft M \& Mosekilde L. Cohort study of risk of fracture before and after surgery for primary hyperparathyroidism. BMJ 2000 321 598-602. (doi:10.1136/bmj.321.7261.598)

23 Khosla S, Melton LJ III, Wermers RA, Crowson CS, O'Fallon Wm \& Riggs Bl. Primary hyperparathyroidism and the risk of fracture: a population-based study. Journal of Bone and Mineral Research 1999 14 1700-1707. (doi:10.1359/jbmr.1999.14.10.1700)

24 Romagnoli E, Cipriani C, Nofroni I, Castro C, Angelozzi M, Scarpiello A, Pepe J, Diacinti D, Piemonte S, Carnevale V et al. Bone. "Trabecular Bone Score" (TBS): an indirect measure of bone micro-architecture in postmenopausal patients with primary hyperparathyroidism. Bone 201353 15425-15429. (doi:10.1016/j.bone.2012.11.041)

25 Shapey IM, Jaunoo SS, Hanson C, Jaunoo SR, Thrush S \& Munro A. Primary hyperparathyroidism: how many cases are being missed? Annals of the Royal College of Surgeons of England 201193 294-296. (doi:10.1308/003588411X13020175704352)

Received 17 June 2014

Revised version received 9 July 2014

Accepted 11 July 2014 